\title{
Expression of Krüppel-like factor 9 in breast cancer patients and its effect on prognosis
}

\author{
ZIRONG JIANG, ZHIPING XU, TINGHUI HU, BIN SONG, FENG LI and KAIYIN WANG \\ Department of Surgical Oncology, Ningde Hospital Affiliated to \\ Fujian Medical University, Ningde, Fujian 352100, P.R. China
}

Received June 27, 2019; Accepted January 13, 2020

DOI: $10.3892 / \mathrm{ol} .2020 .11689$

\begin{abstract}
Expression of Krüppel-like factor 9 (KLF9) in breast cancer tissue and its influence on prognosis was investigated. Sixty-eight patients with breast cancer admitted in Ningde Hospital Affiliated to Fujian Medical University from February 2014 to August 2015 were collected, and the expression level of KLF9 in cancerous tissue $(n=68)$ and normal tissue $(n=68)$ of the patients was measured by quantitative real-time PCR (RT-qPCR). The relationship between the expression and clinical pathological features and prognosis of patients was analyzed. The expression level of KLF9 in cancerous tissue was significantly lower than that in normal tissue $(\mathrm{P}<0.05)$. The expression in breast cancer tissue was not significantly correlated with age, height, menstrual status, lymph node metastasis or pathological differentiation $(P>0.05)$, but was significantly correlated with tumor size and clinical stage $(\mathrm{P}<0.05)$. The 1-, 2-, and 3-year survival rates in the high expression group were significantly higher than those in the low expression group $(\mathrm{P}<0.001)$. Univariate Cox regression analysis was carried out according to the 3-year survival of the patients, and the results showed that tumor size $(\mathrm{P}=0.009)$, lymph node metastasis $(\mathrm{P}=0.002)$, pathological differentiation $(\mathrm{P}=0.015)$, clinical stage $(\mathrm{P}=0.013)$, and KLF9 $(\mathrm{P}=0.018)$ were factors affecting the survival of breast cancer patients. Subsequently, multivariate Cox regression analysis of the indicators with differences showed that those indicators were independent predictors of survival of breast cancer patients. In conclusion, KLF9 expression is low in breast cancer tissue, and its expression level is related to tumor size and clinical stage. Moreover, tumor size $>5 \mathrm{~cm}$, lymph node metastasis, low pathological differentiation, high clinical stage and low expression of KLF9 are all important factors that cause death of patients.
\end{abstract}

Correspondence to: Dr Kaiyin Wang, Department of Surgical Oncology, Ningde Hospital Affiliated to Fujian Medical University, 13 Mindong Middle Road, Ningde, Fujian 352100, P.R. China E-mail:kidb38@163.com; 278337703@qq.com

Key words: Krüppel-like factor 9, breast cancer, prognosis, clinicopathology

\section{Introduction}

Breast cancer is the most common malignant tumor among women worldwide and the second leading cause of tumorrelated death (1). The latest data show that 1,735,350 new cancer cases and 609,640 new cancer deaths are expected in the United States by 2018 (2). In recent years, the incidence rate has been increasing and the age of patients tends to be younger (3). Without timely treatment, breast cancer easily metastasize to heart, brain, lung and other important organs, posing a serious threat to human life and health (4). The survival rate of breast cancer patients has improved due to the continuous progress of surgical treatment, radiotherapy and chemotherapy and the wide application of molecular targeting and immunotherapy, but there are still approximately 20-30\% of patients who relapse and develop distant metastasis (5-7). The study of biomarkers can predict the risk and metastasis of malignant tumors, which is helpful for the treatment of breast cancer (8). Therefore, finding molecular markers that affect the prognosis of breast cancer is of important clinical significance.

In recent years, molecular markers of breast cancer have become a hot topic in clinical research. It has been suggested that KRT19 and CEACAM5 can be used as prognostic indicators for breast cancer patients (9). MMP-2 was reported to help predict the occurrence of metastasis and death in breast cancer patients (10). Krüppel-like transcription factor family (KLFs), as transcription factors with zinc finger domain, play an important role in growth and progression of tumors $(11,12)$. Krüppel-like factor 9 (KLF9), one of the important members of KLFs family (13), is highly-conserved and widely expressed in higher mammals and human tissue $(14,15)$. Studies believe that KLFs are involved in the occurrence and progression of various malignant tumors and regulate the proliferation and differentiation of tumor cells. Their dynamic expression is basically consistent with the change of malignant degree of tumors $(16,17)$. KLF9 inhibits multiple signal pathways in glioblastoma stemness. For example, it inhibits tumor growth by suppressing the ITGA6 in integrin pathway (18). The expression of KLF9 was decreased in hepatocellular carcinoma, and its overexpression inhibits migration and proliferation of hepatoma cells and accelerates apoptosis (19). According to a previous study (20), KLF9 may be an inhibitor of invasive growth of breast cancer, and the expression level 
of KLF9 in 'early' invasive cell populations, several publicly expressed data sets and clinical breast cancer samples are also significantly lower than that in normal tissue. However, there are few studies on KLF9 expression in breast cancer tissue and its correlation with prognosis.

Therefore, this study explored the relationship between KLF9 and pathological features and prognosis of patients by detecting expression of KLF9 in normal tissue and breast cancer tissue.

\section{Patients and methods}

General data. Sixty-eight breast cancer patients admitted to Ningde Hospital Affiliated to Fujian Medical University (Ningde, China) from February 2014 to August 2015 were selected, aged $53.17 \pm 12.56$ years, with height of $159.38 \pm 4.57 \mathrm{~cm}$. Twenty cases were poorly and moderately differentiated, and 48 cases were highly differentiated. There were 35 cases at clinical stage I-II and 33 cases at stage III-IV. The specific data are shown in Table I. All patients were pathologically diagnosed with breast cancer. Cancerous tissue $(n=68)$ and normal tissue $(n=68)$ of patients were obtained as specimens. The normal tissue was adjacent normal tissue and referred to the tissue $3 \mathrm{~cm}$ away from the lesion, and there was no invasion of cancer cells.

Inclusion criteria: no harmful habits, such as smoking and drinking; complete clinical and pathological data; Karnofsky score $\geq 60$ points; clear consciousness and cooperative; undergoing liver and kidney function, tumor markers, blood routine and urine routine examinations within two weeks before surgery.

Exclusion criteria: liver dysfunction, severe organ lesions; autoimmune diseases; pregnant or lactating women; mental diseases, consciousness disorders and communication disorders.

This study was approved by the Ethics Committee of Ningde Hospital Affiliated to Fujian Medical University, and a detailed description of the experimental contents was explaned to the patients. Complete informed consent forms were signed.

Detection of KLF9 mRNA expression by quantitative real-time $P C R(R T-q P C R)$. The embedded tissue was cut into sections with a thickness of $20 \mu \mathrm{m}$. Total RNAs were extracted with TRIzol kit (Applied Biosystems; Thermo Fisher Scientific, Inc.) according to the operation instructions. The concentration and purity of RNAs were determined by a spectrophotometer (Shanghai Mapada Instruments Co., Ltd.) after dissolved with diethyl pyrocarbonate (DEPC) treated water. When A260/A280 >1.8, the subsequent reactions were carried out. cDNAs were prepared using cDNA reverse transcription kit (Invitrogen; Thermo Fisher Scientific, Inc.). Some were stored in a refrigerator at $-80^{\circ} \mathrm{C}$ and the others were treated with RT-qPCR (RT-qPCR instrument: ABI7500 Fast; and SYBR Green RT-qPCR kit were from Applied Biosystems; Thermo Fisher Scientific, Inc.) with a $20-\mu 1$ reaction system in total. Amplification conditions: predenaturation at $94^{\circ} \mathrm{C}$ for $30 \mathrm{sec}$, denaturation at $95^{\circ} \mathrm{C}$ for $5 \mathrm{sec}$, then $60^{\circ} \mathrm{C}$ for $34 \mathrm{sec}$, for a total of 40 cycles. Each sample was tested in 3 repeated wells, and the experiment was repeated 3 times
Table I. General data of breast cancer patients (S)/[n (\%)].

\begin{tabular}{lc}
\hline Factors & $\begin{array}{c}\text { Breast cancer patients } \\
(\mathrm{n}=68)\end{array}$ \\
\hline Age (years) & $53.17 \pm 12.56$ \\
Height $(\mathrm{cm})$ & $159.38 \pm 4.57$ \\
Weight $(\mathrm{kg})$ & $50.26 \pm 10.54$ \\
Menstrual state & \\
Premenopause & $29(42.6)$ \\
Postmenopause & $39(57.4)$ \\
Tumor size & \\
$>5$ cm & $15(22.1)$ \\
$\leq 5$ cm & $53(77.9)$ \\
Lymph node metastasis & \\
Yes & $31(45.6)$ \\
No & $37(54.4)$ \\
Pathological differentiation & \\
Poor and moderate & $20(29.4)$ \\
High & $48(70.6)$ \\
Clinical stage & \\
I-II & $35(51.5)$ \\
III-IV & $33(48.5)$ \\
\hline
\end{tabular}

Table II. Comparison of KLF9 expression between cancerous tissues and normal tissues (mean $\pm \mathrm{SD}$ ).

\begin{tabular}{lcc}
\hline Tissue & Cases & KLF9 \\
\hline Cancerous tissue & 68 & $0.78 \pm 0.31$ \\
Normal tissue & 68 & $3.88 \pm 1.07$ \\
t value & & 22.95 \\
P-value & & $<0.001$ \\
\hline
\end{tabular}

KLF9, Krüppel-like factor 9.

independently. The above operations were strictly carried out in accordance with the instructions. In this study, GAPDH was used as an internal reference, and the GAPDH primer was synthesized by Shanghai Sangon Bioengineering Co., Ltd. Forward primer: 5'-CTGGGCTACACTGACACC-3', reverse primer: 5'-AAGTGGGTTGAGGCAATG-3'. The upstream primer and downstream primer of KLF9 were produced by Guangzhou RiboBio Co., Ltd. Forward primer: 5'-GGGAAACCTCCGAAAA-3', reverse primer: 5'-CGTTCACCTGTATGCACTGTA-3'. The results were expressed with relative quantitative method, and the relative expression of the target gene in cancerous tissue and normal tissue was expressed by $2^{-\Delta \mathrm{Ct}}$, where $\Delta \mathrm{Ct}=\left(\mathrm{Ct}_{\mathrm{KLF} 9}-\mathrm{Ct}_{\mathrm{GAPDH}}\right)$.

Follow-up and outcome measures. Patients were followed up by hospital review and by telephone. The survival time of patients was recorded 1 year, 2 years and 3 years after discharge. The difference of KLF9 expression level in 
Table III. Relationship between KLF9 and pathological features (mean \pm SD).

\begin{tabular}{|c|c|c|c|c|}
\hline Factors & Cases & KLF9 mRNA & $\mathrm{t}$ value & P-value \\
\hline Age & & & 0.176 & 0.861 \\
\hline$>45$ years & 42 & $0.77 \pm 0.44$ & & \\
\hline$\leq 45$ years & 26 & $0.79 \pm 0.39$ & & \\
\hline Height & & & 0.088 & 0.930 \\
\hline$>160 \mathrm{~cm}$ & 36 & $0.76 \pm 0.48$ & & \\
\hline$\leq 160 \mathrm{~cm}$ & 32 & $0.77 \pm 0.45$ & & \\
\hline Menstrual status & & & 0.569 & 0.571 \\
\hline Premenopause & 29 & $0.73 \pm 0.51$ & & \\
\hline Postmenopause & 39 & $0.79 \pm 0.36$ & & \\
\hline Tumor size & & & 2.483 & 0.016 \\
\hline$>5 \mathrm{~cm}$ & 15 & $0.44 \pm 0.13$ & & \\
\hline$\leq 5 \mathrm{~cm}$ & 53 & $0.81 \pm 0.57$ & & \\
\hline Lymph node metastasis & & & 0.402 & 0.689 \\
\hline Yes & 31 & $0.74 \pm 0.45$ & & \\
\hline No & 37 & $0.78 \pm 0.37$ & & \\
\hline Pathological differentiation & & & 1.494 & 0.140 \\
\hline Poor and moderate & 20 & $0.68 \pm 0.38$ & & \\
\hline High & 48 & $0.82 \pm 0.34$ & & \\
\hline Clinical stage & & & 5.158 & $<0.001$ \\
\hline I-II & 35 & $0.84 \pm 0.52$ & & \\
\hline III-IV & 33 & $0.35 \pm 0.17$ & & \\
\hline
\end{tabular}

KLF9, Krüppel-like factor 9.

cancerous tissue and normal tissue of breast cancer patients was observed. According to the clinicopathological features, the relationship between KLF9 and the pathological features, prognosis was analyzed. The independent factors affecting the survival of breast cancer was explored.

Statistical processing. SPSS 19.0 Software System (IBM, SPSS) was used to perform statistical analysis on the experimental data. The counting data were expressed as [n (\%)], and Chi-square test was used to carry out inter-group comparison. The measurement data were expressed by mean \pm SD, and the comparison between the two groups was carried out by paired t-test. Survival analysis was carried out with Kaplan-Meier and checked by Log-rank test. Cox regression was used to analyze the factors affecting survival of breast cancer patients, and $\mathrm{P}<0.05$ was considered to indicate a statistically significant difference.

\section{Results}

Comparison of KLF9 expression between cancerous tissue and normal tissue. The expression level of KLF9 in cancerous tissue $(0.78 \pm 0.31)$ of breast cancer patients was significantly lower than that in normal tissue $(3.88 \pm 1.07)$, and the difference was statistically significant $(\mathrm{P}<0.05)$ (Table II).

Relationship between pathological features and KLF9 in cancer tissue. Expression of KLF9 in breast cancer

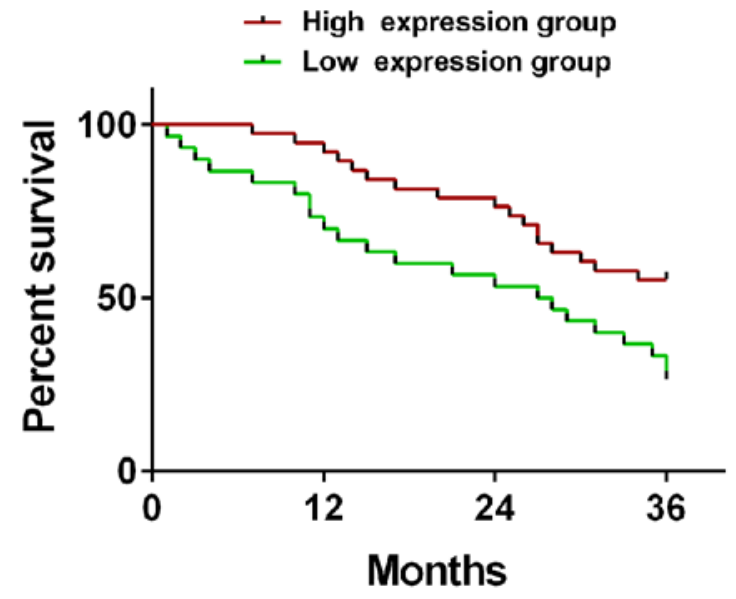

Figure 1. Relationship between KLF9 and survival rate of patients. The patients were divided into high expression group $(\geq 0.78)$ and low expression group $(<0.78)$ according to the expression level of KLF9. Survival analysis was carried out with Kaplan-Meier and checked by log-rank test. The 1-, 2-, and 3-year survival rates in the high expression group were significantly higher than those in the low expression group $(\mathrm{t}=15.72,11.55,16.21 ; \mathrm{P}<0.001)$. KLF9, Krüppel-like factor 9.

tissue showed no significant correlation with age, height, menstrual status, lymph node metastasis or pathological differentiation $(\mathrm{P}>0.05)$. However, it was significantly correlated with tumor size and clinical stage $(\mathrm{P}<0.05)$ as shown Table III. 
Table IV. Univariate analysis of patient survival [n (\%)].

\begin{tabular}{|c|c|c|c|c|}
\hline Factors & Death group $(n=39)$ & Survival group $(\mathrm{n}=29)$ & t value & P-value \\
\hline Age & & & 0.212 & 0.646 \\
\hline$>45$ years & $25(64.1)$ & $17(58.6)$ & & \\
\hline$\leq 45$ years & $14(35.9)$ & $12(41.4)$ & & \\
\hline Height & & & 0.101 & 0.751 \\
\hline$>160 \mathrm{~cm}$ & $20(51.3)$ & $16(55.2)$ & & \\
\hline$\leq 160 \mathrm{~cm}$ & $19(48.7)$ & $13(44.8)$ & & \\
\hline Menstrual status & & & 1.378 & 0.240 \\
\hline Premenopause & $19(48.7)$ & $10(34.5)$ & & \\
\hline Postmenopause & $20(51.3)$ & $19(65.5)$ & & \\
\hline Tumor size & & & 6.761 & 0.009 \\
\hline$>5 \mathrm{~cm}$ & $13(33.3)$ & $2(6.9)$ & & \\
\hline$\leq 5 \mathrm{~cm}$ & $26(66.7)$ & $27(93.1)$ & & \\
\hline Lymph node metastasis & & & 9.379 & 0.002 \\
\hline Yes & $24(61.5)$ & $7(24.1)$ & & \\
\hline No & $15(38.5)$ & $22(75.9)$ & & \\
\hline Pathological differentiation & & & 5.941 & 0.015 \\
\hline Poor and moderate & $16(41.0)$ & $4(13.8)$ & & \\
\hline High & $23(59.0)$ & $25(86.2)$ & & \\
\hline Clinical stage & & & 6.196 & 0.013 \\
\hline I-II & $15(38.5)$ & $20(69.0)$ & & \\
\hline III-IV & $24(61.5)$ & $9(31.0)$ & & \\
\hline KLF9 & & & 5.605 & 0.018 \\
\hline High expression & $17(43.6)$ & $21(72.4)$ & & \\
\hline Low expression & $22(56.4)$ & $8(27.6)$ & & \\
\hline
\end{tabular}

Relationship between KLF9 and survival rate of patients. The patients were divided into high expression group $(\geq 0.78)$ and low expression group $(<0.78)$ according to the expression level of KLF9. The 1-, 2-, and 3-year survival rates of patients in the high expression group $(n=38)$ were $92.1,76.3$, and $55.3 \%$, respectively, while those in the low expression group $(n=30)$ were $70.0,53.3$, and $26.7 \%$, respectively. Therefore, the 1-, 2-, and 3 -year survival rates in the high expression group were significantly higher than those in the low expression group $(\mathrm{t}=15.72,11.55,16.21 ; \mathrm{P}<0.001)$ (Fig. 1).

Cox regression analysis on survival and death of breast cancer. Univariate Cox regression analysis on collected 3-year survival showed that tumor size $(\mathrm{P}=0.009)$, lymph node metastasis $(\mathrm{P}=0.002)$, pathological differentiation $(\mathrm{P}=0.015)$, clinical stage $(\mathrm{P}=0.013)$ and KLF9 $(\mathrm{P}=0.018)$ were the factors affecting the survival of breast cancer patients. Multivariate Cox regression analysis was carried out, and tumor size $>5 \mathrm{~cm}$, lymph node metastasis, low pathological differentiation, high clinical stage and low expression of KLF9 were important factors causing death of patients $(\mathrm{P}<0.05)$, as shown in Tables IV, $\mathrm{V}$ and VI.

\section{Discussion}

Breast cancer is a malignant tumor type that is more common in women (21). The main cause is that breast epithelial cells
Table V. Assignments.

\begin{tabular}{ll}
\hline Factors & \multicolumn{1}{c}{ Assignment } \\
\hline Tumor size & $>5 \mathrm{~cm}: 1 ; \leq 5 \mathrm{~cm}: 2$ \\
Lymph node metastasis & Yes: 1, No: 2 \\
Pathological differentiation & Poor and moderate: 1 , High: 2 \\
Clinical stage & I-II: 1 ; III-IV: 2 \\
KLF9 & High expression $(\geq 0.78): 1$, \\
& low expression $(<0.78): 2$ \\
\hline
\end{tabular}

KLF9, Krüppel-like factor 9.

proliferate abnormally under the stimulation of various internal and external carcinogenic factors, thus exceeding the limit of self-repair and leading to carcinogenesis (22). Its main manifestation is abnormal proliferation of cancer cells, which destroys surrounding normal tissue and changes the normal structure of the breast. At present, there is no effective method to cure breast cancer clinically. In order to achieve early diagnosis and treatment of breast cancer and improve the survival rate, it is of great significance to find biomarkers for its prognosis (23). 
Table VI. Multivariate analysis of factors affecting survival.

\begin{tabular}{lccccc}
\hline Factors & $\beta$ & SD & $\chi^{2}$ value & P-value & HR (95\% CI) \\
\hline Tumor size & 1.849 & 0.933 & 3.927 & 0.048 & $6.354(1.020-39.565)$ \\
Lymph node metastasis & 2.062 & 0.727 & 8.048 & 0.005 & $7.862(1.892-32.679)$ \\
Pathological differentiation & 2.359 & 0.883 & 7.141 & 0.008 & $10.576(1.875-59.647)$ \\
Clinical stage & -1.508 & 0.687 & 4.817 & 0.028 & $0.221(0.058-0.851)$ \\
KLF9 & -1.727 & 0.748 & 5.325 & 0.021 & $0.178(0.041-0.771)$ \\
\hline
\end{tabular}

KLF9, Krüppel-like factor 9; SD, standard deviation; HR, hazards ratio; CI, confidence interval.

KLF9 has been found to have a low expression in endometrial cancer and colorectal cancer $(24,25)$. It regulates genes mainly by binding DNA with its 3 highly conserved and classical Cys2/His2 zinc finger structures at carboxyl terminal (26). KLF9 inhibits Notch1 promoter directly indicated by chromatin immunoprecipitation (ChIP) assay and luciferase reporter gene assay. Moreover, it also regulates neurosphere cells in human glioblastomas by binding to Notch1 promoter and inhibiting Notch1 expression and downstream signal transduction, indicating that KLF9 has differentiation and tumor inhibition functions in tumor initiating cells (27). Moreover, a study has shown that KLF9 inhibits the migration and invasion of breast cancer cells. It is negatively correlated with the expression of matrix metalloproteinase-9 (MMP9), so it inhibits the invasion of cancer cells by downregulating the expression and activity of MMP9 (28).

In this study, the results of RT-qPCR showed that the KLF9 expression in cancerous tissue of breast cancer patients was significantly lower than that in normal tissue. However, some studies have shown that KLF9 has low expression in liver cancer (29) and prostate cancer (30). Hosseini et al (31) found that KLF9 has low expression in infiltrating cell lines and breast cancer tissue, which is consistent with the results of this study. These results indicate that KLF9 plays the role of tumor suppressor gene in the occurrence and progression of tumors. The results of a previous study showed that KLF9 inhibits cancer cell invasion, which was confirmed through differences in expression between invasive and noninvasive cell line, implicating the interaction between proliferation and invasion; the results provide the first evidence that KLF9 is an invasive growth inhibitor of breast cancer (20). In this study, the relationship between KLF9 and pathological features of breast cancer patients was studied, and it was found that the expression of KLF9 in cancerous tissue was not significantly correlated with age, height, menstrual status, lymph node metastasis and pathological differentiation of patients $(\mathrm{P}>0.05)$, but was significantly correlated with tumor size and clinical stage $(\mathrm{P}<0.05)$. It indicates that the production of tumor inhibits the expression of KLF9, and the expression of KLF9 decreases gradually with the severity of the disease. The research of Chen et al (32) showed that KLF9 is correlated with lymph node metastasis, peritoneal effusion and clinical stage of ovarian cancer. The lower the KLF9 level, the more serious the disease is, lymph node metastasis is more likely, and the amount of peritoneal effusion increases. The expression of KLF9 in pancreatic cancer is closely related to the tumor differentiation and vascular invasion, but has no correlation with gender, age, tumor location, TNM stage, nerve invasion and lymph node metastasis (33), suggesting that KLF9 can reflect the conditions of a variety of disease. Limame et al (20) demonstrated that KLF9 is significantly downregulated in breast cancer compared with that in normal breast epithelium, and its expression has no correlation with estrogen receptor (ER), progesterone receptor (PR) or human epidermal growth factor receptor-2 (HER-2). The results show that the downregulation of the expression of this transcription factor is receptor-independent in malignant transformation of breast epithelial cells. The survival analysis in this study showed that the 1-, 2-, and 3-year survival rates in KLF9 high expression group were significantly higher than those in the low expression group. The reason may be that KLF9 inhibits cell proliferation (31). Therefore, it is speculated that KLF9 expression is downregulated in breast cancer and may be closely related to poor prognosis, suggesting that KLF9 can be used to predict the survival of breast cancer patients. Multivariate Cox regression analysis found that tumor size $>5$ $\mathrm{cm}$, lymph node metastasis, low pathological differentiation and high clinical stage are all important factors that cause death of breast cancer patients. Blanchette et al (34) found that HER-2 status, number at first recurrence and metastasis to organs, number of lymph node metastasis and treatment methods are independent prognostic factors for patients with recurrent and metastatic breast cancer. Moreover, a study showed that tumor size and lymph node metastasis number were independent risk factors affecting the prognosis of breast cancer patients (35). This study found that low expression of KLF9 mRNA was one of the independent prognostic indicators of breast cancer, which is similar to the results of Chen et al (32) on KLF9 in the prognosis of ovarian cancer. However, the mechanism of KLF9 in the occurrence and progression of breast cancer needs to be further studied.

This study comprehensively expounded the expression of KLF9 in breast cancer and its relationship with prognosis. It provides a good biomarker for the progression of breast cancer. qPCR measurement has high sensitivity, wide linear range of detection, good detection accuracy and good repeatability. Moreover, the detection cost is low, and it is easy to operate.

Collectively, according to the results of RT-qPCR, KLF9 expression in breast cancer tissue, and its expression level is low related to tumor size and clinical stage. Moreover, tumor 
size $>5 \mathrm{~cm}$, lymph node metastasis, low pathological differentiation, high clinical stage and low expression of KLF9 are all important factors that cause death of patients.

\section{Acknowledgements}

Not applicable.

\section{Funding}

No funding was received.

\section{Availability of data and materials}

The datasets used and/or analyzed during the present study are available from the corresponding author on reasonable request.

\section{Authors' contributions}

ZJ conceived the study and wrote the manuscript. ZX analyzed and interpreted the patient general data. ZX, ZJ, TH and BS performed PCR. FL and KW were responsible for observation indicators analysis. All authors read and approved the final manuscript.

\section{Ethics approval and consent to participate}

The study was approved by the Ethics Committee of Ningde Hospital Affiliated to Fujian Medical University (Ningde, China). Patients who participated in this research had complete clinical data. Signed informed consents were obtained from the patients and/or the guardians.

\section{Patient consent for publication}

Not applicable.

\section{Competing interests}

The authors declare that they have no competing interests.

\section{References}

1. Torre LA, Siegel RL, Ward EM and Jemal A: Global cancer incidence and mortality rates and trends - an update. Cancer Epidemiol Biomarkers Prev 25: 16-27, 2016.

2. Taitt HE: Global Trends and prostate cancer: A Review of incidence, detection, and mortality as influenced by race, ethnicity, and geographic location. Am J Men Health 12: 1807-1823, 2018

3. Curado MP, Oliveira MM, Silva DRM and Souza DLB: Epidemiology of multiple myeloma in 17 Latin American countries: An update. Cancer Med 7: 2101-2108, 2018.

4. Boloker G, Wang C and Zhang J: Updated statistics of lung and bronchus cancer in United States (2018). J Thorac Dis 10: 1158-1161, 2018.

5. Leithner D, Horvat JV, Ochoa-Albiztegui RE, Thakur S, Wengert G, Morris EA, Helbich TH and Pinker K: Imaging and the completion of the omics paradigm in breast cancer. Radiologe 58 (Suppl 1): 7-13, 2018

6. Suppan C and Balic M: Early stage breast cancer treatment and prognostic factors: Post San Antonio Breast Cancer Symposium 2016. Memo 10: 82-85, 2017.
7. Early Breast Cancer Trialists' Collaborative Group (EBCTCG): Effects of chemotherapy and hormonal therapy for early breast cancer on recurrence and 15-year survival: An overview of the randomised trials. Lancet 365: 1687-1717, 2005.

8. Feng Y, Spezia M, Huang S, Yuan C, Zeng Z, Zhang L, Ji X, Liu W, Huang B, Luo W, et al: Breast cancer development and progression: Risk factors, cancer stem cells, signaling pathways, genomics, and molecular pathogenesis. Genes Dis 5: 77-106, 2018.

9. Wang XM, Zhang Z, Pan LH, Cao XC and Xiao C: KRT19 and CEACAM5 mRNA-marked circulated tumor cells indicate unfavorable prognosis of breast cancer patients. Breast Cancer Res Treat 174: 375-385, 2019.

10. Ramos EAS, Silva CT, Manica GC, Pereira IT, Klassen LM, Ribeiro EM, Cavalli IJ, Braun-Prado K,Lima RS, Urban CA, et al: Worse prognosis in breast cancer patients can be predicted by immunohistochemical analysis of positive MMP-2 and negative estrogen and progesterone receptors. Rev Assoc Med Bras (1992) 62: 774-781, 2016.

11. Gao Y, Cao Q, Lu L, Zhang X, Zhang Z, Dong X, Jia W and Cao Y: Kruppel-like factor family genes are expressed during Xenopus embryogenesis and involved in germ layer formation and body axis patterning. Dev Dyn 244: 1328-1346, 2015.

12. Kerr K, Qualmann K, Esquenazi Y, Hagan J and Kim DH: Familial syndromes involving meningiomas provide mechanistic insight into sporadic disease. Neurosurgery 83: 1107-1118, 2018.

13. Cho N: Molecular subtypes and imaging phenotypes of breast cancer. Ultrasonography 35: 281-288, 2016.

14. Apara A, Galvao J, Wang Y, Blackmore M, Trillo A, Iwao K, Brown DP Jr, Fernandes KA, Huang A, Nguyen T, et al: KLF9 and JNK3 interact to suppress axon regeneration in the adult CNS. J Neurosci 37: 9632-9644, 2017.

15. Yılmaz TU, Trabzonlu L, Güler SA, Baran MA, Pösteki G, Erçin $\mathrm{C}$ and Utkan Z: Characteristics of special type breast tumors in our center. Eur J Breast Health 14: 17-22, 2018.

16. Song JL, Chen C, Yuan JP and Sun SR: Progress in the clinical detection of heterogeneity in breast cancer. Cancer Med 5: 3475-3488, 2016.

17. Noguchi K, Eguchi H, Konno M, Kawamoto K, Nishida N, Koseki J, Wada H, Marubashi S, Nagano H, Doki Y, et al: Susceptibility of pancreatic cancer stem cells to reprogramming. Cancer Sci 106: 1182-1187, 2015.

18. Ying M, Tilghman J, Wei Y, Guerrero-Cazares H, QuinonesHinojosa A, Ji H and Laterra J: Kruppel-like factor-9 (KLF9) inhibits glioblastoma stemness through global transcription repression and integrin $\alpha 6$ inhibition. J Biol Chem 289: 32742-32756, 2014.

19. Fu DZ, Cheng Y, He H, Liu HY and Liu YF: The fate of Krüppel-like factor 9-positive hepatic carcinoma cells may be determined by the programmed cell death protein 5 . Int $\mathrm{J}$ Oncol 44: 153-160, 2014

20. Limame R, de Beeck KO, Van Laere S, Croes L, De Wilde A, Dirix L, Van Camp G, Peeters M, De Wever O, Lardon F, et al: Expression profiling of migrated and invaded breast cancer cells predicts early metastatic relapse and reveals Krüppel-like factor 9 as a potential suppressor of invasive growth in breast cancer. Oncoscience 1: 69-81, 2013.

21. Menyhárt O, Fekete JT and Györffy B: Demographic shift disproportionately increases cancer burden in an aging nation: Current and expected incidence and mortality in Hungary up to 2030. Clin Epidemiol 10: 1093-1108, 2018.

22. Vangangelt KMH, van Pelt GW, Engels CC, Putter H, Liefers GJ, Smit VTHBM, Tollenaar RAEM, Kuppen PJK and Mesker WE: Prognostic value of tumor-stroma ratio combined with the immune status of tumors in invasive breast carcinoma. Breast Cancer Res Treat 168: 601-612, 2018.

23. Dieci MV, Vernaci G and Guarneri V: Escalation and de-escalation in HER2 positive early breast cancer. Curr Opin Oncol 31: 35-42, 2019.

24. Kang L, Lü B, Xu J, Hu H and Lai M: Downregulation of Krüppel-like factor 9 in human colorectal cancer. Pathol Int 58: 334-338, 2008.

25. Wang J, Galvao J, Beach KM, Luo W, Urrutia RA, Goldberg JL and Otteson DC: Novel roles and mechanism for Krüppel-like factor 16 (KLF16) regulation of neurite outgrowth and ephrin receptor A5 (EphA5) expression in retinal ganglion cells. J Biol Chem 291: 18084-18095, 2016.

26. Kang L and Lai MD: BTEB/KLF9 and its transcriptional regulation. Yi Chuan 29: 515-522, 2007 (In Chinese). 
27. Ying M, Sang Y,Li Y, Guerrero-Cazares H, Quinones-Hinojosa A, Vescovi AL, Eberhart CG, Xia S and Laterra J: Krüppel-like family of transcription factor 9, a differentiation-associated transcription factor, suppresses Notch1 signaling and inhibits glioblastoma-initiating stem cells. Stem Cells 29: 20-31, 2011.

28. Bai XY, Li S, Wang M, Li X, Yang Y, Xu Z, Li B, Li Y, Xia K, Chen $\mathrm{H}$, et al: Krüppel-like factor 9 down-regulates matrix metalloproteinase 9 transcription and suppresses human breast cancer invasion. Cancer Lett 412: 224-235, 2018.

29. Sun J, Wang B, Liu Y, Zhang L, Ma A, Yang Z, Ji Y and Liu Y: Transcription factor KLF9 suppresses the growth of hepatocellular carcinoma cells in vivo and positively regulates p53 expression. Cancer Lett 355: 25-33, 2014.

30. Shen P, Sun J, Xu G, Zhang L, Yang Z, Xia S, Wang Y, Liu Y and Shi G: KLF9, a transcription factor induced in flutamide-caused cell apoptosis, inhibits AKT activation and suppresses tumor growth of prostate cancer cells. Prostate 74: 946-958, 2014.

31. Hosseini MK, Gunel T, Gumusoglu E, Benian A and Aydinli K: MicroRNA expression profiling in placenta and maternal plasma in early pregnancy loss. Mol Med Rep 17: 4941-4952, 2018.
32. Chen Y, Liu X, Li Y, Quan C, Zheng L and Huang K: Lung cancer therapy targeting histone methylation: opportunities and challenges. Comput Struct Biotechnol J 16: 211-223, 2018

33. Xie VK, Li Z, Yan Y, Jia Z, Zuo X, Ju Z, Wang J, Du J, Xie D, Xie K, et al: DNA-methyltransferase 1 induces dedifferentiation of pancreatic cancer cells through silencing of Krüppel-Like factor 4 expression. Clin Cancer Res 23: 5585-5597, 2017.

34. Blanchette PS, Desautels DN, Pond GR, Bartlett JMS Nofech-Mozes S, Yaffe MJ and Pritchard KI: Factors influencing survival among patients with HER2-positive metastatic breast cancer treated with trastuzumab. Breast Cancer Res Treat 170: 169-177, 2018.

35. Xu T, Li D, He Y, Zhang F, Qiao M and Chen Y: The expression level of CSDAP1 in lung cancer and its clinical significance. Oncol Lett 16: 4361-4366, 2018.

(i)(3) This work is licensed under a Creative Commons Attribution-NonCommercial-NoDerivatives 4.0 International (CC BY-NC-ND 4.0) License. 